\title{
Scale-free networks without growth
}

\author{
Yan-Bo Xie, Tao Zhou, and Bing-Hong Wang* \\ Department of Modern Physics and Nonlinear Science Center and, \\ University of Science and Technology of China, Hefei, 230026, PR China
}

(Dated: November 7, 2018)

\begin{abstract}
In this letter, we proposed an ungrowing scale-free network model, wherein the total number of nodes is fixed and the evolution of network structure is driven by a rewiring process only. In spite of the idiographic form of $G$, by using a two-order master equation, we obtain the analytic solution of degree distribution in stable state of the network evolution under the condition that the selection probability $G$ in rewiring process only depends on nodes' degrees. A particular kind of the present networks with $G$ linearly correlated with degree is studied in detail. The analysis and simulations show that the degree distributions of these networks can varying from the Possion form to the power-law form with the decrease of a free parameter $\alpha$, indicating the growth may not be a necessary condition of the self-organizaton of a network in a scale-free structure.
\end{abstract}

PACS numbers: 89.75.Hc, 64.60.Ak, 84.35.+i, 05.40.-a

Many social, biological, and communication systems can be properly described as complex networks with nodes representing individuals and edges mimicking the interactions among them [1, 2, 3]. Examples are numerous: these include the Internet, the World Wide Web, social networks, metabolic networks, brain networks, food webs, and many others. Recent empirical studies indicate that the networks in various fields have some common characteristics, the most important of which are called small-world effect [4] and scale-free property [5]. The ubiquity of complex networks and the discoveries of the common network properties inspire scientists to construct general models. In the simplest way, these models can be categorized into two classes, the growing models and the ungrowing ones. In the growing models, the number of nodes in network grows continuously, while in ungrowing cases, the number of nodes is fixed. The Watts-Strogatz (WS) model is the pioneer and representative one of ungrowing models, which can be constructed by starting with a regular network and randomly moving one endpoint of each edge with probability $p$ [4]. WS networks display small-world effect, that is, they are highly clustered and of small average distance. The most successful one of growing models is the Barabási-Albert (BA) model [5], which suggests that two main ingredients of the self-organization of a network in a scale-free structure are growth and preferential attachment. These points to the facts that most networks grow continuously by adding new nodes, which are preferentially attached to existing nodes with a large number of neighbors.

By using the mean-field theory on a toy model, Barabási et al assert that the growth is one of the necessary conditions for the emergence of scale-free property [6], and the networks without growth may display an exponential degree distribution. This hypothesis is now widely accepted. However, some real-life networks

*Electronic address: bhwang@ustc.edu.cn, Fax:+86-551-3603574. having fixed size (or almost fixed size) are of scale-free property. For instance, consider the friendship networks of school children wherein the students are represented by nodes and two nodes are connected by an edge if the corresponding students have good friendship [7, 8]. These networks do not grow with time since the number of students in a class is almost fixed. Some students have many good friends, while many others have only a few friends. The students' heterogeneous societal ability leads to a skewed degree distribution (or a skewed strength distribution for weighted case [8]), approximated to a powerlaw form. A typical example in biological systems is the functional networks of human brain [9]. Although its structure varies with time, the brain functional network's sizes is unchanged [10] while it displays scale-free property. Another significant examples are the relationship networks of countries like world trade web 11, 12 and world exchange arrangements web [13, 14]. These networks are scale-free, while their sizes almost do not change in the recent a few years.

Although these networks mentioned above have (almost) fixed size, they are not static since their structures vary with time. These motions can be considered as rewiring processes, that is, some existing edges are removed while some new ones are added. For example, in friendship networks, good friends may quarrel about beliefs, money, or some other things, and become impassive to each other; while some ones will become good friends for their common interests and difficulties. The economic globalization promotes different countries' economies to be integrated together in terms of trade and capital flow, making countries more interdependent than ever. In order to avoid financial crises, more flexible economic regime should be implemented, and the government may change the exchange-rate/trade regime for certain economic and political reasons, leading redistribution of degree/weight in world exchange arrangement networks and world trade networks. These redistribution/rewiring processes make the structures varying ceaselessly in recent years, but the scale-free prop- 


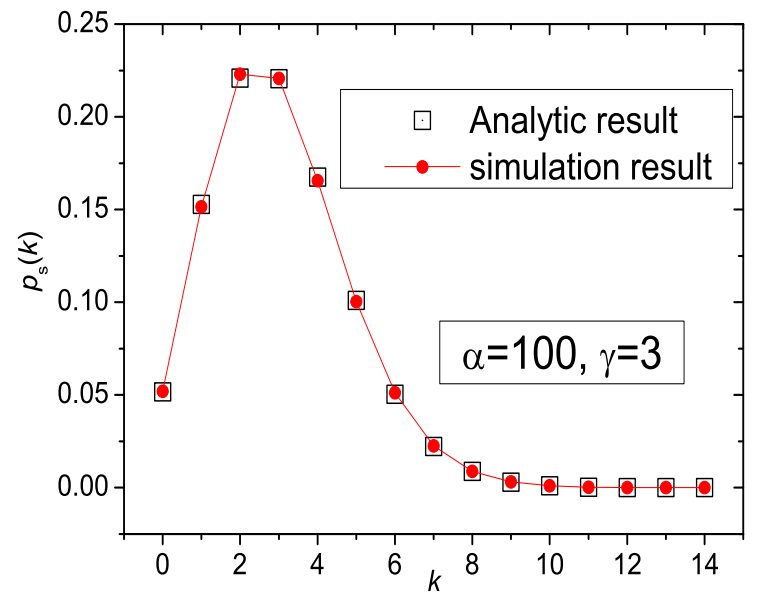

FIG. 1: (Color online) The degree distribution of the present model for the case $\alpha \gg 1$. The black hollow squares and red solid circles represent the analytic and simulation results, respectively. The analytic result agrees with the simulation accurately, and both of them obey Possion forms.

erty is always observed. Notice that, in the ungrowing model proposed by Barabási et al [6] , no edges will be removed thus no rewiring processes will occur. In this letter, for the first time to our knowledge, a scale-free network model without growth is proposed, and we argue that the ungrowing networks can have power-law degree distributions, which attributes to the rewiring processes.

For simplicity, we consider a network model with both fixed number of nodes $N$ and fixed number of edges $E$. The initial state of the network can be a random graph or any other types of graph. Then the network evolves based on the following rewiring processes: At each time step, an edge is randomly selected and removed from the network. At the same time, a node is selected with the preferential probability $G\left(k_{i}\right)$, where $k_{i}$ is the degree of the $i$ th node. Another node is selected also with the above preferential probability. Then a new edge connecting these two nodes is created. Notice that in this rewiring process, the total number of edges is unchanged. The above process is repeated at each time step. Finally, we expect that the network reaches an equilibrium state which is independent of the initial state.
Let $P(k, t)$ represents the number of nodes with degree $k$ at the time step $t . P(k, t)$ satisfies the following normalization conditions

$$
\begin{gathered}
\sum_{k} P(k, t)=N, \\
\sum_{k} k P(k, t)=2 E, \\
\sum_{k} G(k) P(k, t)=1 .
\end{gathered}
$$

It is straightforward to write down the master equation for $P(k, t)$,

$$
\begin{aligned}
& P(k, t+1)-P(k, t)=\frac{(k+1) P(k+1, t)-k P(k, t)}{E} \\
& +2 G(k-1) P(k-1, t)-2 G(k) P(k, t) .
\end{aligned}
$$

It may be helpful to explain the physical meaning of various terms in the above equation. The first two terms on the right hand side represent the net gain of $P(k, t)$ due to the edge removing process. In particular, the first term represents the net gain of $P(k, t)$ when the removing edge connects a node with the degree $k+1$. Notice that when an edge is randomly selected, the connecting nodes have more chance to be of large degree. Explicitly, the node with degree $k$ is selected with the probability $k / \sum_{k} k P(k, t)=k / 2 E$. Since each edge connects to two nodes, the first term is thus $(k+1) P(k+1, t) / E$. Similarly, the second term represents the net loss of $P(k, t)$ when the removing edge connects a node with the degree $k$. The third and fourth terms represents the net gain of $P(k, t)$ due to the edge adding process. The third term represents the net gain of $P(k, t)$ when the adding edge connects a node with the degree $k-1$. Notice that a node with degree $k$ is selected with the probability $G(k)$. The fourth term represents the net loss of $P(k, t)$ when the adding edge connects a node with the degree $k$.

When $t$ is sufficiently large, we expect that $P(k, t)$ approaches a stationary state denoted by $P_{s}(k)$ that satisfies

$$
\frac{(k+1) P_{s}(k+1)-k P_{s}(k)}{2 E}+G(k-1) P_{s}(k-1)-G(k) P_{s}(k)=0,
$$

where $P_{s}(k)$ also satisfy the normalization conditions Eqs.(1-3). Notice that this master equation is a second order equation in which $P_{s}(k+1)$ is determined by $P_{s}(k)$ and $P_{s}(k-1)$. Eqs. $(1-3,5)$ can be solved by the following 
method. Define

$$
H(k)=G(k) P_{s}(k)
$$

and

$$
W(k)=k P_{s}(k) / 2 E .
$$

Then Eqs. $(2-3,5)$ can be rewritten as

$$
\begin{gathered}
\sum_{k} W(k)=1, \\
\sum_{k} H(k)=1, \\
H(k)=H(k-1)+W(k+1)-W(k) .
\end{gathered}
$$

By iteration, one can obtain

$$
H(k)=H(0)+W(k+1)-W(1) .
$$

Since $W(0)=0$ and

$$
\sum_{k} H(k)=\sum_{k} W(k)=1,
$$

one immediately have

$$
W(k+1)=H(k)
$$

for $k \geq 0$. Substituting Eqs.(6-7) into Eq.(12), one obtains the following solution

$$
P_{s}(k)=\frac{(2 E)^{k}}{k !} G(k-1) G(k-2) \ldots G(0) P_{s}(0)
$$

for $k \geq 1$ with $P_{s}(0)$ determined by the normalization condition Eq.(1). It should be stressed that given the dependence of $G(k)$ on $k$, there is still a proportional coefficient in $G(k)$ determined by Eq.(3).

Hereinafter, we focus on a special form, also one of the simplest cases, of $G(k)$, where $G(k)$ is linearly correlated with $k$,

$$
G(k)=\frac{k+\alpha}{\sum_{i=1}^{N}\left(k_{i}+\alpha\right)}=\frac{k+\alpha}{2 E+N \alpha},
$$

where $k_{i}$ denotes the degree of node $i$, and $\alpha$ is a constant (Notice that $G(k)$ satisfies the normalization condition Eq. (3).). Substituting the above equation into Eq. (13), one can straightforwardly obtain, in this special case, that

$$
\begin{gathered}
P_{s}(0)=N\left(\frac{\alpha}{\alpha+\gamma}\right)^{\alpha} \\
P_{s}(k)=N\left(\frac{\alpha}{\alpha+\gamma}\right)^{\alpha}\left(\frac{\gamma}{\alpha+\gamma}\right)^{k} \frac{1}{k !} \alpha(\alpha+1) \ldots(\alpha+k-1), k \geq 1
\end{gathered}
$$

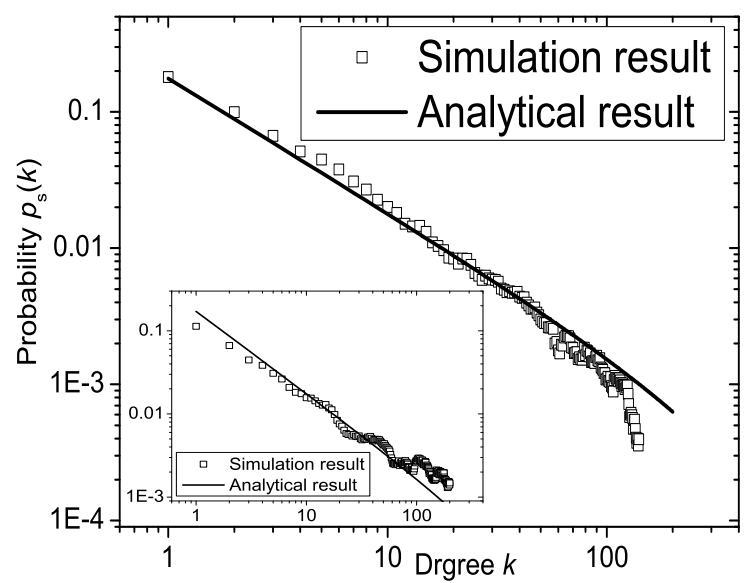

FIG. 2: The degree distribution of the present model with $\alpha=0.01$ and $\gamma=5$. The hollow squares and solid curve represent the simulation and analytical results, respectively. The analytical result agrees with the simulation well, obeying an approximately power-law form. The inset exhibits the case of $\alpha=0.01$ and $\gamma=10$ for comparison.

where $\gamma=2 E / N$.

When $\alpha \gg 1$, the mechanism of preferential attachment is destroyed, that is, since $\alpha$ probably plays the main part in the sum $\alpha+k_{i}$, the probability a high degree node being selected is approximately the same as that of a low degree node (see Eq. 14). Therefore, one expects that the present network will approach a completely random network with its degree distribution obeying the Possion distribution 15]

$$
P_{s}(k)=N \frac{\mathrm{e}^{-\gamma}}{k !} \gamma^{k} .
$$

When $\alpha \ll 1$ and $\gamma \ll 1$, most nodes are isolated and the nodes with large degree are very few. However, when $\alpha \ll 1$ and $\gamma \succeq 1$, interesting result emerges. The corresponding degree distribution obeys a power-law form with exponent approximated to 1 when $k \ll \gamma / \alpha$

$$
P_{s}(k)=N\left(\frac{\alpha}{\gamma}\right)^{\alpha} \frac{\alpha}{k} .
$$

In succession, we will show some simulations. $10^{6}$ time steps are performed in all simulations while only the final $2 \times 10^{5}$ time steps are used for statistical average. The initial graph is simply the completely random graph with given $N$ and $E$. The network size if fixed as $N=1000$ unless a special statement is addressed. In figure 1 , we report the case for very large $\alpha$, where $p_{s}(k):=P_{s}(k) / N$ denotes the normalized probability function for degree distribution. The simulation result strongly supports the analytic one (see Eq. (16)), and both of them obey Possion forms. In this figure, one will find that there are about $5 \%$ nodes with degree zero. However, this probability only reflects the average number of isolated nodes 


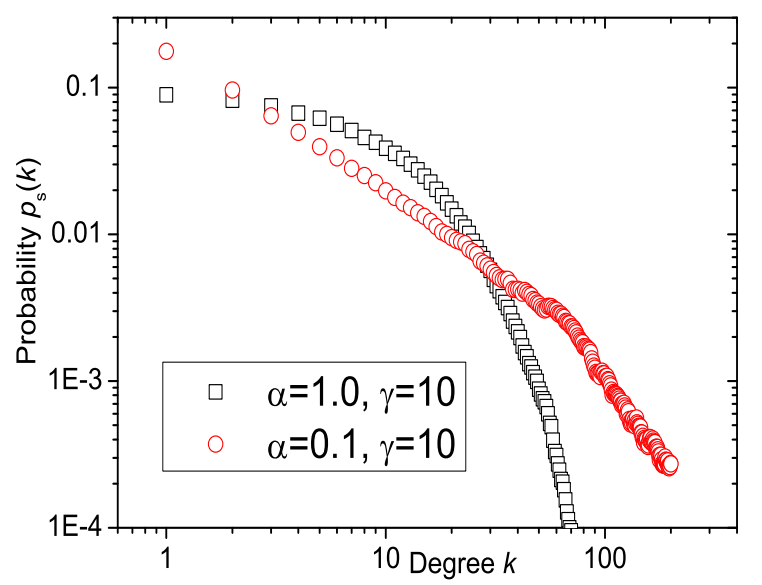

FIG. 3: (Color online) The degree distributions of the present model. The parameter $\gamma=10$ is fixed, and the black squares and red circles represent the cases with $\alpha=1.0$ and $\alpha=$ 0.1 , respectively. The degree distribution with $\alpha=1.0$ obeys approximately an exponential form rather than a power-law form.

over all configurations. Because of the rewiring mechanism, no nodes are always isolated. Although real-life networks are not always connected, most previous studies have focused on connected graphs. Therefore, hereinafter, we neglect the isolated nodes.

In figure 2, we report the analytical and simulation results about degree distributions generated by very small $\alpha=0.01$ and $\gamma=5$. The degree distribution displays obviously scale-free property with a cut-off at $k \approx 120$. The solid curve denotes the analytical solution, which agrees with the simulation well. We also have checked that the departure from Eq.(18) will get greater if the parameter $\alpha$ becomes larger. In the inset, the case of $\gamma=10$ is shown for comparison. As mentioned above, the two extreme cases with $\alpha \approx 0$ and $\alpha \gg 1$ will lead to the power-law and Possion distributions, respectively. In figure 3, we report the simulation results for $\alpha=0.1$ and $\alpha=1.0$. The departure from the power law becomes larger as the increase of $\alpha$, and the degree distribution with $\alpha=1.0$ obeys approximately an exponential form rather than a power-law form.

In conclusion, we proposed an ungrowing model and obtained the analytic results for arbitrary forms of selection probability $G(k)$. One special kind of the present networks is investigated in detail, which can generate networks with different classes of degree distributions by tuning two parameters. Especially, when $\alpha$ is close to zero and $\gamma \succeq 1$, the present networks display scale-free property with its exponent approximately equal to that of world exchange arrangement web [14].

The previous empirical studies have demonstrated the extensive existence of scale-free networks. Most of them are growing at all times, while some others are of (almost) fixed size. Most previous models [1, 2, 3, 5, 6] suggested the growth a key ingredients of the emergence of scale-free structure, thus fail to provide an underlying mechanism by which the ungrowing networks exhibiting scale-free property. Here we argue that the rewiring mechanism widely existed in real-life networks like friendship networks 7, 8], brain functional networks 9], world trade web [11, 12], and world exchange arrangements web [13, 14], may be the origin of the emergence of scale-free structure in fixed-size networks.

Finally, we would like to point out that many different classes of degree distributions can be obtained from different forms of $G(k)$ [16]. In particular, one can obtain the power law degree distribution $P_{s}(k) \sim k^{-\beta-1}$ with tunable exponent $\beta$ by setting $G(k) \sim \frac{k^{\beta+1}}{(k+1)^{\beta}}$ when the number of edges $E$ is properly chosen [16].

This work is support by the National Natural Science Foundation of China under Nos. 70271070, 10472116, 70471033, and 70571074, and the Specialized Research Fund for the Doctoral Program of Higher Education under No. 20020358009.
[1] R. Albert and A. -L. Barabási, Rev. Mod. Phys. 74, 47(2002).

[2] S. N. Dorogovtsev and J. F. F. Mendes, Adv. Phys. 51, 1079(2002).

[3] M. E. J. Newman, SIAM Review 45, 167(2003).

[4] D. J. Watts and S. H. Strogatz, Nature 393, 440(1998).

[5] A. -L. Barabási and R. Albert, Science 286, 509(1999).

[6] A. -L. Barabási, R. Albert, and H. Jeong, Physica A 272, 173(1999).

[7] A. Rapoport, and W. J. Horvath, Behav. Sci. 6, 279(1961).

[8] B. Hu, X. -Y. Jiang, J. -F. Ding, Y. -B. Xie, and B. -H. Wang, Physica A 353, 576(2005).

[9] V. M. Eguíluz, D. R. Chialvo, G. A. Cecchi, M. Baliki, and A. V. Apkarian, Phys. Rev. Lett. 94, 018102(2005).
[10] In human brain functional networks, each node represents a voxels of dimension $3 \times 3.475 \times 3.475 \mathrm{~mm}^{3}$ in the picture taken by functional magnetic resonance imaging (FMRI) in humans. Therefore, the network size is fixed as $N=36 \times 64 \times 64$.

[11] M. A. Serrano, and M. Boguñá, Phys. Rev. E 68, 015101(2003).

[12] X. Li, Y. -Y. Jin, and G. Chen, Physica A 328 287(2003).

[13] M. Mussa, P. Masson, A. Swoboda, E. Jadresic, P. Mauro, and A. Berg, Exchange Rate Regimes in an Increasing Integrated World Economy, International Monetary Fund, Washington DC, 2002.

[14] X. Li, Y. -Y. Jin, and G. Chen, Physica A 343 573(2004).

[15] B. Bollobás, Random Graphs (Academic Press, New York, 2001). 
[16] Y. -B. Xie, T. Zhou, and B. -H. Wang, to be published. 